\title{
TEMPORAL ADVERBIALS AS ELEMENTS OF THE VERBAL CODE REPRESENTING THE MNEMONIC FUNCTION
}

\author{
Irina V. Tivyaeva \\ Tula State University, Tula, Russia
}

\begin{abstract}
Accepting W. Chafe's ideas regarding memory's representability via language categories expressed in grammatical forms, the author aims at analyzing temporal adverbials as grammatical markers of mnemonic processes, establishing their status in this quality and assessing their potential. To provide a thorough and accurate description of temporal adverbials consistent with the goal of the present study, two relevant parameters are set: direct involvement in marking of specific mnemonic processes at the grammatical level and the type of temporal reference assigned to the mnemonic event.

In accordance with the temporal characteristic of the mnemonic event, two subclasses of temporal adverbials are differentiated: localizers specifying the position of the mnemonic event on the time axis in relation to the fixed or conditional reference point and correlators indicating the position of the mnemonic event in relation to other events.

An analysis of communicative contexts representing interaction between the subject of a mnemonic situation with other participants yielded results indicating absence of correlation between subclasses of temporal adverbials and specific mnemonic processes as well as functional inequality as representation of mnemonic function at the grammatical level.

The findings suggest involvement of temporal adverbials in setting the timeline framework of a mnemonic utterance regardless of their discretionary status and validity as grammatical markers of mnemonic function in general as opposed to specific memory types and procedures.

Key words: memory, temporal adverbial, mnemonic process, mnemonic situation, mnemonic utterance.

Citation. Tivyaeva I.V. Temporal Adverbials As Elements of the Verbal Code Representing the Mnemonic Function. Vestnik Volgogradskogo gosudarstvennogo universiteta. Seriya 2, Yazykoznanie [Science Journal of Volgograd State University. Linguistics], 2018, vol. 17, no. 1, pp. 124-133. (in Russian). DOI: https://doi.org/ 10.15688/jvolsu2.2018.1.13
\end{abstract}

\section{ТЕМПОРАЛЬНЫЕ АДВЕРБИАЛЫ КАК ЭЛЕМЕНТ ВЕРБАЛЬНОГО КОДИРОВАНИЯ МНЕМИЧЕСКОЙ ДЕЯТЕЛЬНОСТИ}

\author{
Ирина Владимировна Тивьяева \\ Тульский государственный университет, г. Тула, Россия
}

\begin{abstract}
Аннотация. Основываясь на идеях У. Чейфа о принципиальной репрезентируемости памяти языковыми категориями, воплощающимися в грамматических структурах, автор ставит цель охарактеризовать ста$\infty$ тус и потенциал темпоральных адвербиалов как грамматических маркеров мнемической деятельности. В ка군 честе параметров, релевантных для описания темпоральных адвербиалов в данной функции, выделяются ๓ं участие в маркировании конкретных процессов памяти и способ темпоральной характеристики мнемического события.

В соответствии с типом временно́го ориентира дифференцированы два класса темпоральных адвербиалов: локализаторы, определяющие положение мнемического события на оси времени относительно фиксированного или условного ориентира, и корреляторы, локализующие мнемическое событие относительно другого события. В результате анализа контекстов, репрезентирующих коммуникативное взаимо-
\end{abstract}


действие англоязычных участников мнемической ситуации, установлено, что выделенные классы темпоральных адвербиалов не связаны корреляционными отношениями с мнемическими процессами, при этом возможности отдельных классов рассматриваемых единиц как грамматических маркеров процессов памяти неодинаковы.

Полученные результаты свидетельствуют о том, что темпоральные адвербиалы принимают участие в формировании темпорального плана мнемического высказывания, не являясь его облигаторным компонентом, и могут рассматриваться как грамматические маркеры индивидуальной мнемической деятельности в целом, а не отдельных процессов или видов памяти.

Ключевые слова: память, темпоральный адвербиал, мнемический процесс, мнемическая ситуация, мнемическое высказывание.

Цитирование. Тивьяева И. В. Темпоральные адвербиалы как элемент вербального кодирования мнемической деятельности // Вестник Волгоградского государственного университета. Серия 2, Языкознание. 2018. - T. 17, № 1. - C. 124-133. - DOI: https://doi.org/10.15688/jvolsu2.2018.1.13

\section{Введение}

Изучение «репрезентации ментальных структур в вербальных произведениях» входит в круг актуальных проблем отечественного языкознания, которое, как верно отмечает В.А. Пищальникова, объективно движется «в сторону психолингвистической и когнитивной парадигм» [Пищальникова, 2004, c. 161]. Предпринятое нами исследование посвящено одной из конкретных задач в рамках указанного направления, а именно выявлению и описанию специфики репрезентации процессов памяти на грамматическом уровне.

Проблема взаимоотношения памяти и языка в равной степени интересует и психологов, и языковедов. При этом как в лингвистических, так и в психологических исследованиях мнемической деятельности в качестве вербальной формы фиксации и трансляции памяти традиционно рассматриваются две языковые единицы: лексема и текст (см., например, [Переходцева, 2012; Попова, Егорова, 2015; Ребрина, 2008; Центнер, 2012; Erll, 2011]). Изучение лексики, номинирующей мнемические процессы, и мемориальных текстов, в первую очередь нарративов, позволяет приблизиться к пониманию принципов функционирования единого механизма памяти в ее индивидуальном и надындивидуальном измерении.

Между тем известно, что мнемическая деятельность субъекта манифестируется в языке не только на лексическом и текстовом уровнях. Она способна проявляться и в грамматических категориях, на что первым из лингвистов указал У. Чейф, убедительно продемонстрировав, что при изложении событий прошлого выбор позиции обстоятельства времени в английском предложении определяется когнитивным контекстом, а именно принадлежностью информационного актанта к определенному типу памяти: «speakers must have some cognitive basis for making the discrete choice between weak and strong temporal adverbs» [Chafe, 1973, p. 272] «выбор говорящим сильного или слабого темпорального адвербиала определяется некоторым когнитивным основанием» (здесь и далее перевод наш. - И. Т.). Он подробно описал, какие инференции формируются у коммуниканта в процессе интерпретации сообщения о событии из прошлого в зависимости от наличия / отсутствия ориентиров, локализирующих событие во времени, и их позиции в высказывании. По У. Чейфу, в английском языке грамматически кодируются три вида памяти: поверхностная (surface), кратковременная (shallow) и долговременная (deep). Их сигналами на уровне высказывания выступают темпоральные адвербиалы (temporal adverbs): сильные (strong), занимающие инициальную позицию в предложении, и слабые (weak), занимающие финальную позицию. При этом отсутствие темпорального адвербиала также является значимым и интерпретируется как маркер поверхностной памяти. Анализируя различные высказывания, вербализующие прошлый опыт субъекта, и их инференции, У. Чейф приходит к следующему заключению: «material from deep memory must be reported with a strong adverb, that material from shallow memory may be reported with either a strong or a weak adverb, 
and that material from surface memory may be reported with a strong adverb, a weak adverb, or no adverb at all» [Chafe, 1973, p. 272] - «при вербализации содержимого долговременной памяти используется сильный адвербиал, при вербализации содержимого кратковременной памяти могут использоваться как сильные, так и слабые адвербиалы, при вербализации содержимого поверхностной памяти адвербиалы могут отсутствовать или могут использоваться и сильные, и слабые адвербиалы».

Бесспорным достоинством концепции У. Чейфа является подтверждение принципиальной репрезентируемости памяти на грамматическом уровне, ограничение же концепции, на наш взгляд, связано с отождествлением ретроспективной памяти, фактически положенной в основу разработанной У. Чейфом типологии памяти и ее языковых маркеров, с памятью индивидуальной, представленной не только воспоминанием, но и процессами помещения, хранения и утраты информации.

В настоящей работе предпринимается попытка преодолеть данное ограничение и определить роль темпоральных адвербиалов в процессе вербального кодирования любой мнемической деятельности англоязычного субъекта. Следует отметить, однако, что в фокусе исследования находятся в первую очередь темпоральные адвербиалы, репрезентирующие «собственно темпоральную адвербиальность» в терминологии Ц.Д. Бидагаевой [Бидагаева, 2011], тогда как адвербиальные единицы, выражающие аспектуальную и таксисную адвербиальность, остались за рамками работы. Данное решение обусловлено главным образом ориентацией автора на устоявшуюся в междисциплинарном пространстве memory studies трактовку категории темпоральности как единства трех измерений настоящего, прошлого и будущего (см., например: [Dalla Barba, 2012]). Таким образом, в статье ставится цель выявить и описать классы темпоральных адвербиалов, участвующих в маркировании различных процессов памяти посредством указания на временну́ю локацию мнемического события, установить их статус в этом качестве и оценить их потенциал как грамматических маркеров мнемической деятельности.

\section{Материал и методика исследования}

В соответствии с поставленной целью исследования был осуществлен сбор эмпирических данных, а именно коммуникативных контекстов, иллюстрирующих взаимодействие англоязычных коммуникантов в «мнемической ситуации», обязательными компонентами которой являются субъект, осуществляющий мнемическую деятельность, вербализуемый процесс памяти и его информационный актант (подробное описание мнемической ситуации как инструмента анализа языковой репрезентации процессов памяти представлено в работах Р.Ф. Исхаковой, Е.Г. Хомяковой [Исхакова, Хомякова, 2009] и Ю.Н. Рогачевой [Рогачева, 2002]).

Источниками фактического материала послужили современные англоязычные художественные и документальные произведения разных жанров, прежде всего, мемуары и автобиографии (источники первого типа), а также тексты сферы публичной, массовой (источники второго типа) и компьютерно опосредованной коммуникации (источники третьего типа). Общее количество текстовых фрагментов, вошедших в эмпирическую базу исследования, составляет 7500 , при этом указанные типы источников представлены в равных долях, то есть на каждый тип источника приходится 2500 коммуникативных фрагментов.

На первом этапе обработки фактических данных отобранные текстовые фрагменты были распределены в соответствии с типом мнемического процесса, манифестируемого в рамках мнемической ситуации (запоминание, хранение, воспоминание и забывание), что позволило выявить возможность темпоральных адвербиалов кодировать процессы памяти.

На втором этапе зарегистрированные в эмпирической базе фрагменты были маркированы как содержащие или не содержащие темпоральные адвербиалы, которые локализуют мнемическое событие на шкале времени. Количество высказываний, содержащих интересующие нас единицы, составило 5097 , что соответствует $67,96 \%$ от общей выборки. Необходимо отметить, что общее количество темпоральных адвербиалов в зарегист- 
рированных фрагментах превышает данное число, так как в границах одного фрагмента нами было зафиксировано до пяти темпоральных адвербиалов.

Отобранные в результате описанной процедуры контексты были проанализированы нами в соответствии со следующими параметрами: 1) способ темпоральной характеристики мнемического события, 2) роль в процессе вербального кодирования мнемической деятельности.

Поскольку класс темпоральных адвербиалов изначально достаточно разнороден и среди лингвистов нет единого мнения относительно его конституентов, мы считаем необходимым уточнить, какие именно единицы мы причисляем к данному классу и какой из его подклассов представляет интерес для нашего исследования. Принимая за основу определение адвербиала Н.А. Слюсаревой, которая характеризует его как единицу, «связанную не только со сказуемыми, но и со всем предложением в целом» [Слюсарева, 1986, c. 116], в данной работе под темпоральным адвербиалом мы понимаем обстоятельство времени, функция которого в предложении не ограничивается указанием временных координат действия, выраженного глаголом, но расширяется до передачи временного фона описываемой предложением ситуации.

Среди исследователей нет единства в определении состава темпоральных адвербиалов. Так, И.М. Богуславский включает в состав темпоральных адвербиалов единицы разных лексико-грамматических категорий, объединенные общей адвербиальной функцией (наречия, частицы, предлоги, союзы) [Богуславский, 1996]. Ц.Д. Бидагаева причисляет к группе темпоральных адвербиалов наречия, наречные слова, предложные группы и субстантивные группы [Бидагаева, 2004]. Дж. Лич и Я. Свартвик относят к темпоральным адвербиалам наречия, предложные группы, субстантивные группы и придаточные времени с подчинительными союзами [Leech, Svartvik, 1983].

При интерпретации результатов, полученных в ходе обработки эмпирических данных, мы опираемся на типологию, предложенную зарубежными лингвистами, как наиболее полную. В качестве темпоральных адвербиалов, маркирующих мнемическую деятельность субъекта, мы рассматриваем наречия, именные и предложные группы, а также придаточные времени, выполняющие обстоятельственную функцию и соотносящие мнемическое событие, кодируемое посредством мнемического высказывания, с некоторым фиксированным или условным временным ориентиром.

\section{Результаты и обсуждение}

В ходе анализа коммуникативных контекстов, входящих в нашу картотеку, с опорой на классификацию Ц.Д. Бидагаевой [Бидагаева, 2004, с. 18-20] было выделено два класса темпоральных адвербиалов в соответствии со способом темпоральной характеристики мнемического события: адвербиалылокализаторы, соотносящие мнемическое событие с некоторым фиксированным или условным временным ориентиром, и адвербиалы-корреляторы, характеризующие мнемическое событие относительно какоголибо другого события.

Адвербиалы-локализаторы представлены двумя типами: абсолютные и относительные.

Абсолютные локализаторы указывают на точное время мнемического события. В качестве таких локализаторов используются наречия и именные группы (с предлогом или без предлога), обозначающие часовое время, дату числом, дату годом, части суток, времена года, дни недели, месяцы, праздники, знаменательные события, возраст, периоды человеческой жизни и пр.

Например:

(1) I recall seeing her first as Nina in The Seagull at the Lyric Hammersmith in 1985 (Billington).

(2) Ultimately, I can't remember everything. But I do remember leaving barefoot at 2am (Anonymous).

Фрагменты (1) и (2) - отрывки из мнемических монологов, вербализующих процессы воспоминания и хранения информации в памяти соответственно. В обоих случаях мнемическое событие, как реконструируемое из памяти, так и удерживаемое в ней, имеет четкую точечную локализацию на временной оси, не зависимую от момента речи и позиции субъекта мнемической ситуации. В примеpe (1) абсолютным временным ориентиром 
является указание на год совершения действия, в примере (2) функцию абсолютного темпорального маркера выполняет указание на часовое время.

Относительные локализаторы представляют собой дейктические маркеры, локализующие мнемическое событие по отношению к говорящему или к моменту речи. Такие локализаторы выражены главным образом наречиями и именными группами, указывающими на краткие или продолжительные временные интервалы, синхронные моменту речи, предшествующие ему или следующие за ним.

(3) I remember as a child that I used to worry about her (Higgins Clark, p. 320).

(4) "The last thing I remember is going out with some friends from work last night" (Kinsella, p. 29-30).

Во фрагментах (3) и (4) мнемический процесс хранения информации в памяти маркируется относительными адвербиалами, а именно локализаторами предшествования. В обоих примерах субъект мнемической ситуации заявляет об удержании в памяти информации о ситуациях в прошлом, положение которых на оси времени относительно условного ориентира, то есть момента говорения, отмечено темпоральными адвербиалами, конкретизирующими возраст говорящего и время суток.

(5) I'll never forget this one (Patterson).

В примере (5) относительный темпоральный адвербиал never выполняет функцию локализатора следования, характеризуя мнемический процесс как направленный в будущее относительно текущего момента.

(6) I still remember the hours I sat at that desk, the Blue Danube softly playing, clicking between the different views from my space ship, watching the stars dart by, watching the empty circle planets rotate. I paid J15 for Elite and another J15 for the joystick. It was so much money to me back then. But while there were other games that looked better and were more exciting, I don't remember many of them now (Stuart).

В мнемическом монологе (6) (структурно-семантические особенности мнемических монологов и других типов мнемического высказывания подробно описаны в: [Тивьяева,
2013]) темпоральные адвербиалы back then и now подчеркивают границу между двумя временными планами - планом воспоминаний и планом настоящего. Now как локализатор синхронности задает точку отсчета в текущем времени, back then отсылает к некоторому временно́му промежутку в прошлом, предшествующему моменту говорения.

Адвербиалы-корреляторы, в отличие от абсолютных и относительных локализаторов, задающих точку или интервал мнемического события или указывающих на его положение относительно фиксированного или условного временно́го ориентира, дают темпоральную характеристику мнемическому событию через отсылку к моменту другого события.

Например,

(7) My earliest cinematic memory is watching a film one afternoon in the mid 1970s with my mother before I started primary school (Gray).

Во фрагменте (7) мнемическое событие, о котором вспоминает говорящий, локализуется во времени посредством абсолютного локализатора in the mid $1970 \mathrm{~s}$, задающего определенный интервал в прошлом, граница которого далее конкретизируется коррелятором предшествования, выраженным придаточным времени before I started primary school. Таким образом, темпоральный коррелятор, уточняющий координаты мнемического события относительно другого действия, служит дополнительным маркером вербализации мнемической деятельности и позволяет локализовать информационный актант процесса памяти с большей точностью.

В следующем высказывании, вербализующем процесс запоминания, мнемическое событие принадлежит сфере планируемого субъектом:

(8) Well, the key I will begin from now on to learn it by heart; it is only four digits and thus no problem, I will memorize it, until I know it by heart, or maybe I will write it on the wall in a corner of the house so I do not lose it. And I will record it inside, in my head. That is the best... to memorize it (Jensen, p. 77).

В приведенном фрагменте мнемическое событие получает двойную темпоральную характеристику, позволяющую с большей точностью определить его место на временно́й 
шкале будущего посредством локализатора следования from now on и коррелятора предшествования until I know it by heart.

(9) "I remember Jimmy when we were in the seventh grade," he wrote (Higgins Clark, p. 182).

В примере (9), иллюстрирующем процесс хранения в памяти, коррелятор синхронности, выраженный придаточным времени, локализует мнемическое событие посредством указания на другое действие, протекавшее параллельно с ним.

(10) < ...> Mommy when I start school and I am scared, God will take my hand and I will remember you. Mommy, when I want to see a ball game I will save a seat. I will remember you. Mommy, when I want to learn to ride a bike and don't know what to do. I will remember you. Mommy, when I want to protect my little sisters and show them what to do, I will remember you. Mommy, I know we have family that care and family that love us, but I will remember you $<$... $>$ (Lang).

В мнемическом монологе (10) корреляторы следования дают темпоральную характеристику процессу хранения информации в памяти, определяя его как непрерывный и перманентный, не имеющий конца в обозримом будущем.

В результате анализа эмпирического материала установлено, что возможности различных конституентов класса темпоральных адвербиалов относительно манифестации мнемических процессов неодинаковы. Так, абсолютные локализаторы были зарегистрированы нами в мнемических высказываниях, репрезентирующих процессы воспоминания и за- бывания, но не были зафиксированы как маркеры запоминания и хранения в памяти, что, на наш взгляд, объясняется, прежде всего, природой мнемических процессов. Ретроспективно направленные процессы воспоминания и забывания, как правило, оперируют информацией об уже свершившихся фактах, которые поддаются абсолютной темпоральной локализации, тогда как мнемическое событие, обрабатываемое процессами хранения и помещения в память, часто ориентировано на текущий момент или на перспективу, и при описании такого события связи с временным ориентиром манифестируются относительными локализаторами или корреляторами.

Максимальным потенциалом при маркировании мнемической деятельности обладают темпоральные корреляторы, поскольку локализации через последующее, синхронное или предшествующее действие поддаются информационные актанты всех процессов памяти, как проспективно, так и ретроспективно ориентированных. Полученные данные, отражающие возможности темпоральных локализаторов и корреляторов в отношении манифестации отдельных процессов памяти, представлены в таблице 1.

Частота встречаемости временных локализаторов и корреляторов как маркеров мнемической деятельности также варьируется в различных типах мнемического высказывания - мнемическое диалогическое единство (МДЕ), мнемический монолог (ММ), мнемический нарратив (МН). Количественные показатели, отражающие употребление темпоральных адвербиалов в МДЕ, ММ и MH, входящих в нашу картотеку, представле-

Таблица 1

Потенциал темпоральных адвербиалов при маркировании различных процессов памяти

\begin{tabular}{|c|c|c|c|c|}
\hline \multirow[b]{2}{*}{$\begin{array}{c}\text { Темпоральные } \\
\text { адвербиалы }\end{array}$} & \multicolumn{4}{|c|}{ Маркируемый мнемический процесс } \\
\hline & $\begin{array}{c}\text { Помещение } \\
\text { информации } \\
\text { в память } \\
\end{array}$ & $\begin{array}{c}\text { Хранение } \\
\text { информации } \\
\text { в памяти } \\
\end{array}$ & $\begin{array}{c}\text { Восстановление } \\
\text { информации } \\
\text { из памяти } \\
\end{array}$ & $\begin{array}{c}\text { Утрата } \\
\text { информации } \\
\text { из памяти } \\
\end{array}$ \\
\hline Локализаторы & - & - & - & - \\
\hline абсолютные & - & - & + & + \\
\hline относительные & - & + & + & + \\
\hline Корреляторы & - & - & - & - \\
\hline предиествование & - & + & + & + \\
\hline одновременность & - & + & + & + \\
\hline следование & + & + & + & + \\
\hline
\end{tabular}


ны в таблице 2. В абсолютном выражении указано количество мнемических высказываний каждого типа, в состав которых входит хотя бы один соответствующий темпоральный адвербиал. Процентные показатели отражают долю мнемических высказываний, содержащих локализатор или коррелятор, среди общего числа мнемических высказываний того же типа.

Минимальные значения были зарегистрированы нами для МДЕ, а максимальные для мнемического нарратива. Такое распределение показателей мы склонны связывать с особенностями формата высказывания-вербализатора. В частности, специфика мнемического нарратива заключается в его объеме, политематичности и политемпоральности, поэтому смещение акцента в сторону той или иной тематики, переход от одного мнемического события к другому, смена темпорального плана требуют дополнительных сигналов, снижающих риск потери или искажения информации при передаче сообщения от нарратора получателю. МДЕ, напротив, не отличается значительным объемом, характеризуется монотематичностью и обладает монотемпоральной или битемпоральной структурой, которая формируется в первую очередь глагольными формами, поэтому в грамматической структуре МДЕ темпоральные адвербиалы играют второстепенную роль.

Преобладание временных корреляторов над локализаторами во всех типах мнемических высказываний мы связываем с их более высоким потенциалом в маркировании отдельных мнемических процессов.

Статус темпоральных адвербиалов как грамматических маркеров мнемической деятельности также подтверждается тем фактом, что зона действия задаваемого ими интервала не ограничивается рамками предло- жения. Полученные результаты позволяют утверждать, что установленный темпоральным адвербиалом временной интервал действителен в пределах мнемического высказывания, за исключением тех случаев, когда он отменяется или модифицируется другим темпоральным адвербиалом.

Рассмотрим следующий пример:

(11) He later explained that the following year, when I was seven, the shit hit the fan. This may explain why I do not have any recollection of that year, save for vague memories of late-night screaming matches and crashings about in the dining room, when my parents returned from the theater. I was usually reading under the covers, and one night a strange stench of alcohol came from the kitchen, which I followed to find my mother pouring a number of bottles of booze down the sink. That year is a blank, aside from my seventh birthday party (I got a splinter in my nose). The next thing I remember comes a year later, when I was abruptly informed that we were moving, without apparent reason, to Minnesota (Hornbacher, p. 33-34).

В отрывке представлены воспоминания нарратора о детстве, верхняя граница которого обозначена относительным темпоральным локализатором the following year в сочетании с коррелятором when I was seven, а нижняя граница - относительным локализатором a year later. Действие заданного временно́го интервала поддерживается дважды повторяющейся лексической единицей year, принадлежащей к тематической группе слов со значением прошлого опыта, выступающих в качестве контекстуальных сигналов мнемической переработки личного опыта.

\section{Заключение}

Многопараметрический анализ мнемических высказываний, репрезентирующих

Таблица 2

Распределение темпоральных адвербиалов по различным типам мнемических высказываний в общей выборке

\begin{tabular}{|c|c|c|c|c|c|c|}
\hline \multirow{2}{*}{$\begin{array}{c}\text { Темпоральные } \\
\text { адвербиалы }\end{array}$} & \multicolumn{2}{|c|}{$\begin{array}{c}\text { Мнемическое } \\
\text { диалогическое единство }\end{array}$} & \multicolumn{2}{|c|}{ Мнемический монолог } & \multicolumn{2}{|c|}{ Мнемический нарратив } \\
\hline & $\begin{array}{c}\text { Кол-во } \\
\text { контекстов }\end{array}$ & $\begin{array}{c}\text { Доля среди } \\
\text { общего } \\
\text { кол-ва МДЕ }\end{array}$ & $\begin{array}{c}\text { Кол-во } \\
\text { контекстов }\end{array}$ & $\begin{array}{c}\text { Доля среди } \\
\text { общего } \\
\text { кол-ва ММ }\end{array}$ & $\begin{array}{c}\text { Кол-во } \\
\text { контекстов }\end{array}$ & $\begin{array}{c}\text { Доля среди } \\
\text { общего } \\
\text { кол-ва МН }\end{array}$ \\
\hline Локализаторь & 289 & $19,67 \%$ & 3799 & $67,98 \%$ & 397 & $89,62 \%$ \\
\hline Корреляторь & 467 & $31,79 \%$ & 4252 & $76,09 \%$ & 402 & $90,74 \%$ \\
\hline
\end{tabular}


мнемическую деятельность субъекта в процессе англоязычной коммуникации, позволяет сделать следующие выводы.

1. Темпоральные адвербиалы принимают участие в формировании темпорального плана мнемического высказывания, указывая на локализацию мнемического события относительно фиксированного или условного ориентира, однако не являются облигаторным компонентом мнемического высказывания.

2. В соответствии с типом временно́го ориентира среди темпоральных адвербиалов, маркирующих мнемическую деятельность, выделяются класс локализаторов и класс корреляторов, причем последние количественно и функционально доминируют, что обусловлено природой манифестируемого мнемического процесса.

3. Классы темпоральных адвербиалов не связаны корреляционными отношениями с определенными мнемическими процессами, а следовательно, темпоральные адвербиалы являются грамматическими маркерами индивидуальной мнемической деятельности в целом, а не отдельных процессов или видов памяти. Данную функцию рассматриваемые единицы приобретают в общем контексте мнемической ситуации, взаимодействуя с языковыми средствами других уровней, участвующими в вербальном кодировании результатов работы механизма индивидуальной памяти.

\section{СПИСОК ЛИТЕРАТУРЫ}

Бидагаева Ц. Д., 2004. Темпоральные адвербиалы в английском предложении: языковой процесс выражения смысла. Улан-Удэ : Изд-во ВСГТУ. $187 \mathrm{c}$.

Бидагаева Ц. Д., 2011. Адвербиальность как категория широкой семантики // Magister Dixit. № 2 . С. 26-33.

Богуславский И. М., 1996. Сфера действия лексических единиц. М. : Школа «Языки русской культуры». $460 \mathrm{c}$.

Исхакова Р. Ф., Хомякова Е. Г., 2009. Память и восприятие: некоторые аспекты языковой репрезентации // Вестник Санкт-Петербургского университета. Серия 9. Филология. Востоковедение. журналистика. Вып. 1, ч. 1. C. $84-89$.

Переходцева О. В., 2012. Концепции памяти в современном западном литературоведении // Вест- ник Пермского университета. Российская и зарубежная филология. Вып. 1 (17). С. 157-164.

Пищальникова В. А., 2004. К проблеме определения языковой способности // Языковое бытие человека и этноса: психолингвистический и когнитивный аспекты / под ред. В. А. Пищальниковой. М. : МГЭИ. Вып. 7. С. 159-169.

Попова Л. Г., Егорова В. О., 2015. О сущности и возможностях установления культурной памяти слов (сопоставительный аспект) // Филологические науки. Вопросы теории и практики. № 9-1 (51). С. 143-145.

Ребрина Л. Н., 2008. Лексические средства репрезентации операций памяти в немецком языке: принципы лингвистического анализа // Вестник Волгоградского государственного университета. Серия 2, Языкознание. № 2. C. $112-118$.

Рогачева Ю. Н., 2002. Специфические черты фрейма «память» в современном английском языке // VI Царскосельские чтения. Университетская наука - образованию России : материалы междунар. конф. СПб. : ЛГОУ им. А.С. Пушкина. С. 115-117.

Слюсарева Н. А., 1986. Проблемы функциональной морфологии современного английского языка. М. : Наука. 216 с.

Тивьяева И. В., 2013. Мнемическое высказывание и процедура его идентификации // Вестник Томского государственного университета. № 372. C. 45-48.

Центнер А. С., 2012. Репрезентация представлений о процессах памяти в немецком языке // Критика и семиотика. Вып. 17. С. 224-232.

Chafe W., 1973. Language and Memory // Language. № 49 (2). P. 261-281.

Dalla Barba G., 2012. Memory, Consciousness and Temporality. N. Y. : Springer Science and Business Media. $224 \mathrm{p}$.

Erll A., 2011. Memory in Culture. Basingstoke : Palgrave Macmillan. 209 p.

Leech G., Svartvik J., 1983. A Communicative Grammar of English. М. : Просвещение. 304 c.

\section{ИСТОЧНИКИ}

Anonymous - Anonymous. A letter to... the friend who raped me// The Guardian. 2015. Aug. 15. URL: https://www.theguardian.com/lifeandstyle/2015/ aug/15/a-letter-to-the-friend-who-raped-me.

Billington - Billington M. Natasha Richardson's death is a tragedy for theater // The Guardian. 2009. Mar. 19. URL: https://www.theguardian.com/ culture/2009/mar/19/natasha-richardson-deathtragedy-theatre. 
Gray-Gray S. My earliest cinematic memory... // The Guardian. URL: https://www.theguardian.com/ notesandqueries/query/0,-204010,00.html.

Higgins Clark - Higgins Clark M. Before I say goodbye. N. Y. : Pocket Books, 2001. 371 p.

Hornbacher - Hornbacher M. Wasted: a memoir of anorexia and bulimia. N. Y. : HarperCollins Publishers, Inc., ebook, 2005. 308 p.

Jensen - Jensen S., Ronsbo H. Histories of Victimhood. Philadelphia : University of Pennsylvania Press, 2014. 280 p.

Kinsella - Kinsella S. Remember Me? N. Y. : Dial Press, $2008.390 \mathrm{p}$.

Lang - Lang A. Fears and Tears. Bloomington : Trafford Publishing, 2014. 48 p.

Patterson-Patterson J., Roughan H. You've Been Warned. URL: http:/www.twirpx.com/file/1839808/.

Stuart - Stuart K. No Man's Sky is Elitie for the $21^{\text {st }}$ century. Pointless? Maybe - but also sublime // The Guardian. 2016. Aug. 19. URL: https:// www.theguardian.com/technology/2016/aug/ 19/no-mans-sky-elite.

\section{REFERENCES}

Bidagaeva Ts.D., 2004. Temporal adverbials in English sentence: linguistic process of expressing ideas. Ulan-Ude, Izd-vo VSGTU. 187 p.

Bidagaeva Ts.D., 2011. Adverbiality as a category of broad semantics. Magister Dixit, no. 2, pp. 26-33.

Boguslavskiy I.M., 1996. The domain of lexical units. Moscow, Yazyki russkoy kultury Publ. 460 p.

Iskhakova R.F., Khomyakova E.G., 2009. Memory and Perception: Some Aspects of Linguistic Representation. Vestnik Sankt-Peterburgskogo universiteta. Seriya 9. Filologiya. Vostokovedenie. Zhurnalistika, vol. 1, part 1, pp. 84-89.

Perekhodtseva O.V., 2012. The concepts of memory in contemporary Western literature studies. Vestnik Permskogo universiteta. Rossiyskaya $i$ zarubezhnaya filologiya [Perm University Herald. Russian and Foreign Philology], no. 1 (17), pp. 157-164.

Pishchalnikova V.A., 2004. On the problem of defining linguistic competence. Pishchalnikova V.A., ed. Yazykovoe bytie cheloveka $i$ etnosa: psikholingvisticheskiy $i$ kognitivnyy aspekty [Linguistic Reality of Man and Ethnos: Psycholinguistic and Cognitive Aspects]. Moscow, MGEI Publ., iss. 7, pp. 159-169.

Popova L.G., Egorova V.O., 2015. On the essence of cultural memory of the words and the possibilities to identify it (comparative aspect)]. Filologicheskie nauki. Voprosy teorii i praktiki
[Philological Sciences. Issues of Theory and Practice], no. 9-1 (51), pp. 143-145.

Rebrina L.N., 2008. Lexical means of representing mnemonic operations in German: principles of linguistic analysis. Vestnik Volgogradskogo gosudarstvennogo universiteta. Seriya 2, Yazykoznaniye [Science Journal of Volgograd State University. Linguistics], no. 2, pp. 112-118.

Rogacheva Yu.N., 2002. Specific Features of Frame "Memory" in contemporary English. VI Tsarskoselskie chteniya. Universitetskaya nauka - obrazovaniyu Rossii: materialy mezhdunar. konf. Saint Petersburg, LGOU im. A.S. Pushkina, pp. 115-117.

Slyusareva N.A., 1986. Problems of modern English functional morphology. Moscow, Nauka Publ. $216 \mathrm{p}$.

Tivyaeva I.V., 2013. Mnemonic utterance and the process of its identification. Vestnik Tomskogo gosudarstvennogo universiteta [Tomsk State University Journal], no. 372, pp. 45-48.

Tsentner A.S., 2012. Representation of viewpoints on mnemonic processes in German. Kritika $i$ semiotika [Critique \& Semiotics], iss. 17, pp. 224-232.

Chafe W., 1973. Language and Memory. Language, no. 49 (2), pp. 261-281.

Dalla Barba G., 2012. Memory, Consciousness and Temporality. New York, Springer Science and Business Media. 224 p.

Erll A., 2011. Memory in Culture. Basingstoke, Palgrave Macmillan. 209 p.

Leech G., Svartvik J., 1983. A Communicative Grammar of English. Moscow, Prosveshhenie Publ. 304 p.

\section{SOURCES}

Anonymous. A letter to ... the friend who raped me. The Guardian, August 15, 2015. URL: https:// www.theguardian.com/lifeandstyle/2015/aug/ 15/a-letter-to-the-friend-who-raped-me.

Billington M. Natasha Richardson's death is a tragedy for theater. The Guardian, March 19, 2009. URL: https://www.theguardian. com/culture/2009/mar/ 19/natasha-richardson-death-tragedy-theatre.

Gray S. My earliest cinematic memory... The Guardian. URL: https://www. theguardian. com/ notesandqueries/query/0,,-204010,00.html.

Higgins Clark M., 2001. Before I say goodbye. New York, Pocket Books. 371 p.

Hornbacher M., 2005. Wasted: a memoir of anorexia and bulimia. HarperCollins Publishers, Inc., ebook. 308 p.

JJensen S., Ronsbo H., 2014. Histories of Victimhood. University of Pennsylvania Press. 280 p. 
И.В. Тивьяева. Темпоральные адвербиалы как элемент вербального кодирования

Kinsella S., 2008. Remember Me? NewYork, Dial Press. $390 \mathrm{p}$.

Lang A., 2014. Fears and Tears. Trafford Publishing. 48 p.

Patterson J., Roughan H. You've Been Warned. URL: http://www.twirpx.com/file/1839808/.
Stuart K. No Man's Sky is Elitie for the $21^{\text {st }}$ century. Pointless? Maybe - but also sublime. The Guardian, August 19, 2016. URL: https:// www.theguardian.com/technology/2016/aug/ 19/no-mans-sky-elite.

\section{Information about the Author}

Irina V. Tivyaeva, Candidate of Sciences (Philology), Associate Professor, Department of Linguistics and Translation Studies, Tula State University, Prosp. Lenina, 92, 300012 Tula, Russia, tivyaeva@yandex.ru, https://orcid.org/0000-0002-6316-784X

\section{Информация об авторе}

Ирина Владимировна Тивьяева, кандидат филологических наук, доцент кафедры лингвистики и перевода, Тульский государственный университет, просп. Ленина, 92, 300012 г. Тула, Россия, tivyaeva@yandex.ru,https://orcid.org/0000-0002-6316-784X 